\title{
El formato organizacional del Plan FinEs en la provincia de La Pampa: entre lo nuevo y lo viejo
}

Mariana Correa

Universidad Nacional de La Pampa, Argentina

profmarianacorrea@gmail.com

\begin{abstract}
Resumen
Este artículo presenta algunos análisis realizados en el marco de un estudio de corte cualitativo, denominado "Las experiencias educativas de estudiantes egresados del Plan de Finalización de Estudios Primarios y Secundarios en instituciones de General Pico y Eduardo Castex (La Pampa). La investigación propone un abordaje inicial de la primera etapa del Plan FinEs (2008-2011) en esta provincia. Aquí se presentan conclusiones vinculadas al análisis de su formato organizacional, con énfasis en tres aspectos: la organización del tiempo, los espacios y los recursos disponibles. Se advierte que, si bien en los documentos ministeriales el FinEs se presenta como una "agenda didáctica flexible", adaptada a las necesidades de jóvenes y adultos, su materialización en las sedes replica rasgos propios del formato tradicional de la escuela secundaria, con pocas variantes.
\end{abstract}

Palabras clave: Plan FinEs, formato organizacional, jóvenes, educación secundaria

\section{The organizational format of the Plan FinEs in the province of La} Pampa: between the new and the old

\begin{abstract}
This paper presents some analyses carried out within the framework of a study of qualitative cutting, called "The educational experiences of students who have graduated from the Plan of Completion of Primary and Secondary Studies at institutions of General Pico and Eduardo Castex (La Pampa). The research proposes an initial approach to the first stage of the Plan (2008-2011) in the province. Here are conclusions related to the analysis of their organizational format, with an emphasis on three aspects: the organization of time, spaces and the resources available. Warns that, while ministerial documents presents the FinEs as a "flexible educational agenda", adapted to the needs of young people and adults; its materialization in the headquarters replicates features of the traditional format of high school, with few variations.
\end{abstract}




\section{Introducción}

En nuestro país, la preocupación por la permanencia y el egreso de los y las jóvenes en las escuelas, si bien tiene su historia, emerge con fuerza a partir de la sanción de la Ley de Educación Nacional (LEN) N²6206. Esta normativa, que extiende la obligatoriedad escolar a toda la educación secundaria, configura un escenario educativo sumamente complejo donde la construcción de ciudadanía y la búsqueda de justicia social se constituyen en propósitos centrales (Tenti Fanfani, 2016). La inclusión de aquellos sujetos "invisibilizados" por algunas de las políticas previas promueve un aumento de la matrícula de las escuelas secundarias de todo el país, hecho que no solo pone en tensión los objetivos y la cultura institucional que históricamente caracterizaron al nivel, sino que muestra, además, las dificultades del sistema formal para responder a las necesidades e intereses de todos los/las jóvenes.

En este contexto, surge en la agenda educativa estatal -junto a otras iniciativas con similares propósitos y alcances- el Plan de Finalización de Estudios Primarios y Secundarios (en adelante Plan FinEs) como una política de alcance nacional destinada a jóvenes y adultos que, por diversos motivos, no lograron finalizar sus estudios primarios y secundarios en el sistema educativo formal. En complemento con el desarrollo de otras políticas tales como la Asignación Universal por Hijo; el Programa Conectar Igualdad; la Ley de Protección Integral de los Derechos de los Niños, Niñas y Adolescentes; la Ley Nacional de Salud Reproductiva y el Programa Progresar, surge para atender/profundizar el componente educativo (formación y capacitación) de lo que el Ministerio de Desarrollo Social de la Nación denominó "Políticas sociales integrales" o "políticas públicas con impacto social".

La lectura de las normativas nacionales y provinciales que regulan el FinEs a nivel nacional y jurisdiccional permite delinear las características salientes de su formato organizacional. En uno de los documentos elaborados por el Ministerio de Educación de la Nación titulado "Plan de Finalización de Estudios Primarios y Secundarios para Jóvenes y Adultos. Presentación y Líneas de Acción 2008-2011", se explicita que el Plan FinEs debe lograr que los jóvenes y adultos lleven a cabo sus experiencias educativas en el marco de un modelo institucional que dé respuestas a las necesidades de la población destinataria. A partir de esta afirmación, surgen interrogantes como los siguientes. ¿En qué medida el formato organizacional, que propusieron las sedes del Programa, dio respuestas a las necesidades de los sujetos destinatarios de la política? ¿El FinEs se presenta como una alternativa innovadora en relación con las experiencias educativas previas de los/as estudiantes en la escuela secundaria?

El artículo se organiza en tres apartados: en el primero se presentan la estructura y rasgos centrales de la política a nivel nacional y jurisdiccional; en el segundo, los objetivos, metodología y enfoque teórico que fundamentan la investigación en torno al programa; para luego, en el tercero, presentar el análisis de las percepciones de los estudiantes egresados acerca de tres elementos que constituyen su formato organizacional: la organización del tiempo, del espacio y la administración de los recursos.

\section{El Plan FinEs en la provincia de La Pampa (2008-2011)}

El FinEs se desarrolla en dos etapas: la primera, iniciada en el año 2008, destinada a aquellos sujetos que finalizaron su educación secundaria sin acreditar espacios 
curriculares correspondientes al último año de escolaridad; y la segunda, a partir de 2012, pensada para jóvenes y adultos que no hubieran transitado la escuela primaria o secundaria en su totalidad (Plesnicar, 2014). Es de destacar que el programa se enmarca en la modalidad de Educación Permanente de Jóvenes y Adultos como un "programa a término", en el seno de una tradición que desde sus inicios promueve experiencias de intersectorialidad y participación social en los procesos educativos y que en los contextos actuales mantiene su lucha por revertir el lugar subalterno que históricamente ocupó en el sistema educativo, producto de su ubicación como "régimen especial" en la reforma neoliberal de la década de los noventa (Finnegan y Brunetto, 2014).

La política -a nivel nacional- se materializa en las instituciones a partir de un modelo de gestión que articula las capacidades y acciones del Ministerio de Educación de la Nación, los Ministerios de Educación de las provincias y ciertas entidades conveniantes (con las que se suscribe un convenio) situadas en los contextos locales en los que el Programa se desarrolle. El modelo puede interpretarse del siguiente modo:

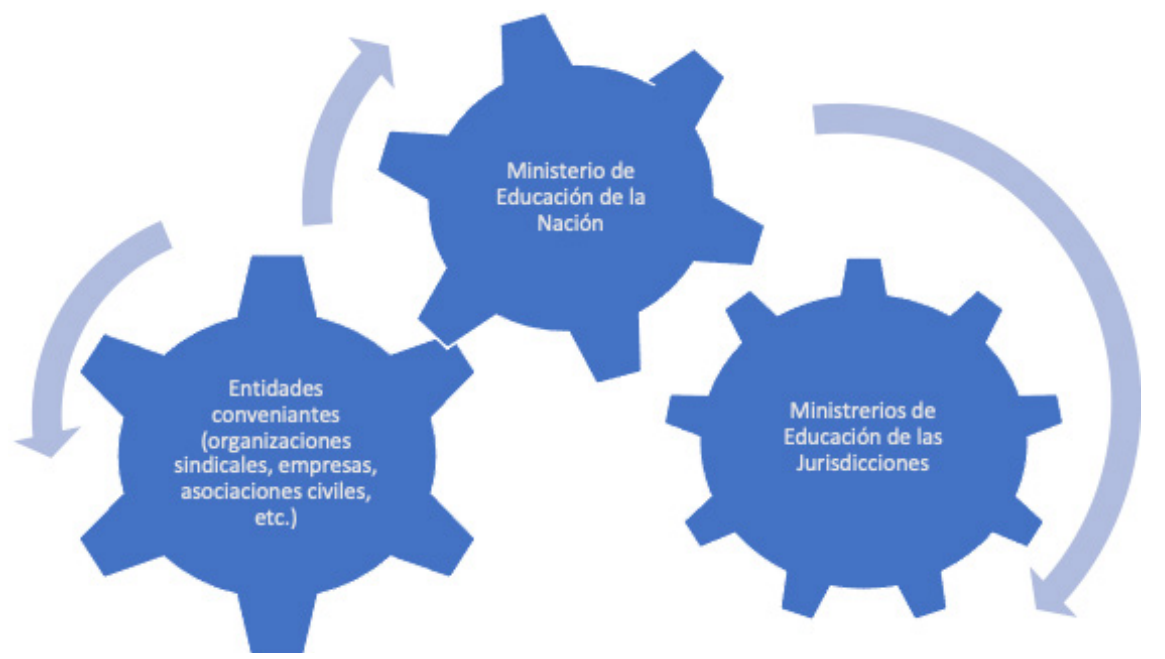

Fuente: Elaboración propia a partir de la lectura de documentos ministeriales nacionales.

La normativa nacional señala que las sedes pueden funcionar en entidades conveniantes, en escuelas secundarias comunes y de educación técnica que adhieran al Programa Nacional de Becas Estudiantiles y en escuelas secundarias de modalidad de jóvenes y adultos (CENS). Este modelo de gestión es replicado en la provincia de La Pampa pero con algunas modificaciones. Por ejemplo, la normativa provincial, en su presentación de la Primera Etapa de la política (2008-2011), omite la inclusión de entidades conveniantes para el desarrollo del Programa y explicita que las sedes solo se ubicarán en instituciones educativas. Así, según datos proporcionados por el sitio oficial del FinEs y en correspondencia con los niveles de concentración poblacional de las distintas localidades de la provincia, la distribución de las sedes se realizó del siguiente modo: 


\begin{tabular}{|l|l|}
\hline \multicolumn{2}{|c|}{ Plan FinEs- Primera Etapa (Período 2008-2011) } \\
\hline \multicolumn{1}{|c|}{ Localidades } & Número de sedes \\
\hline Santa Rosa & 7 \\
\hline General Pico & 1 \\
\hline General Acha & 1 \\
\hline Realicó & 1 \\
\hline Jacinto Arauz & 1 \\
\hline La Maruja & 1 \\
\hline Santa Isabel & 1 \\
\hline Victorica & 1 \\
\hline 25 de Mayo & 1 \\
\hline Anguil & 1 \\
\hline Eduardo Castex & \\
\hline
\end{tabular}

Fuente: Elaboración propia a partir de los datos publicados en www.fines.educ.ar

El programa es presentado y difundido como una "agenda didáctica flexible" donde el docente tutor y el estudiante acuerdan un plan y cronograma de producción de actividades de enseñanza y aprendizaje a lograr en ocho encuentros tutoriales y una evaluación final (Resolución MCE N 962/ 08: Anexo I). Las propuestas de tutorías se conforman a partir de las denominadas "áreas básicas" de conocimiento: Matemática, Lengua y Literatura, Ciencias Sociales, Ciencias Naturales e Inglés. ${ }^{1}$ Señala que los docentes tutores son quienes deben identificar los contenidos del área o las áreas que el estudiante deberá preparar para ser evaluado en el espacio curricular o bien del área que adeuda. En el caso de los llamados "espacios curriculares específicos", no comprendidos en las áreas básicas, explicita que será fundamental su relevamiento por parte de la Secretaría de las sedes para la evaluación de su implementación.

Vale destacar que en la provincia la normativa no explicita los espacios curriculares que incluirán las propuestas de tutorías, pero aclara que entre las responsabilidades de las escuelas de procedencia de los/as estudiantes está la de convocar a los/as alumnos/ as con materias pendientes, según los registros del libro matriz de la institución educativa. ${ }^{2}$ Los documentos ministeriales coinciden en señalar que los docentes tutores son quienes deben seleccionar contenidos de los diversos campos disciplinares para luego elaborar una propuesta pedagógica flexible y un dispositivo para su evaluación. 


\section{Objetivos y construcción teórico-metodológica de la investigación}

Como ya se mencionó, el estudio focaliza su indagación en la primera etapa del Plan FinEs. Desde una perspectiva de investigación cualitativa, propone analizar las experiencias educativas de estudiantes egresados/as de dos sedes del Programa en la provincia de La Pampa: una ubicada en la ciudad de General Pico y la otra situada en la localidad de Eduardo Castex. Para ello, se plantean los siguientes objetivos: describir los sentidos que adquiere para los/las jóvenes la finalización de sus estudios; analizar los modos en que los rasgos organizativos del Plan -la organización del tiempo, el espacio y los recursos- impactan en la construcción de sus experiencias educativas; explorar las implicancias de la propuesta curricular de la política en estas y problematizar las relaciones de los sujetos con el conocimiento en el Nivel Polimodal y en el Programa.

Con referencia al enfoque conceptual del estudio, se adoptaron como herramientas de análisis los contextos de trayectorias o ciclos de las políticas (Policy Cicle Approach) propuestos por Stephen Ball $(1997,2002,2008)$, dispositivos que permiten explorar desde una perspectiva sociológica el desarrollo de las políticas. El autor propone abordarlas a partir de cinco contextos interrelacionados: contexto de influencia, contexto de la producción del texto político, contexto de la práctica, contexto de los resultados y contexto de la estrategia política. Los contextos son definidos como arenas de actividad social y discursiva a través de los cuales las políticas se mueven, son producidas, creadas y ajustadas. No se conciben en forma secuencial o lineal, sino como "anidados" unos dentro de otros (Mainardes y Marcondes, 2009). Vale señalar que en la investigación se abordaron de modo inicial los primeros tres contextos mencionados.

En lo referente a las decisiones metodológicas, se llevó adelante un estudio de caso de tipo exploratorio (Neiman y Quaranta, 2007). ${ }^{3}$ De este modo, se realizaron entrevistas semiestructuradas a una muestra representativa de estudiantes egresados/as de dos sedes del Programa: tal como se anticipó, una ubicada en la ciudad de General Pico, que funciona simultáneamente con otras cinco sedes y otra -única en su contexto- situada en la localidad de Eduardo Castex. Los estudiantes entrevistados egresaron del FinEs en el año 2011, momento en que finalizó la primera etapa del Programa. El análisis de las voces de los actores se complementó con el abordaje de diferentes marcos normativos que regulan el Programa a nivel nacional y provincial.

\section{El formato organizacional del Programa: tiempos, espacios y recursos}

Son múltiples y variados los estudios que advierten la necesidad de problematizar las condiciones organizacionales en las que se llevan adelante prácticas pedagógicas tendientes a la inclusión de los sujetos en la escuela (Baquero, Diker y Frigerio, 2007; Dussel, 2006; Terigi, 2009). Los análisis del "formato" señalan que solo una verdadera modificación de las estructuras organizativas de las instituciones puede generar transformaciones al nivel de las prácticas. Por esto, las políticas educativas que proponen formatos organizacionales alternativos, como lo hace el Plan FinEs, merecen especial atención. 
Una revisión de la literatura reciente permite advertir que el término formato organizacional, a diferencia de "gramática escolar" y de "régimen académico", refiere a aquellas coordenadas que conforman el denominado núcleo duro de la escuela moderna y que aún hoy persisten en su configuración: la organización del currículum por disciplinas, el ciclo y el año escolar como unidad temporal, la organización de los recursos y del trabajo docente (Ziegler, 2011). En esta línea, Flavia Terigi plantea que desde sus comienzos la escuela secundaria se estructuró a partir de tres disposiciones básicas: "la clasificación de los currículos, el principio de designación de los profesores por especialidad, y la organización del trabajo docente por horas de clase" (Terigi, 2007: 64). Estas disposiciones conforman un trípode de hierro, un patrón organizacional que está en la base de buena parte de las críticas al nivel medio y que es muy difícil de modificar.

A los rasgos mencionados, otros autores agregan la simultaneidad, la obligatoriedad, la presencialidad, organizaciones específicas de agrupamientos y espacios escolares, entre otros aspectos (Trilla, 1985; Tiramonti, 2007). En palabras de Myriam Southwell (2010), se trata de un engranaje en el que todos los elementos mencionados están fuertemente imbricados, conformando una unidad.

\section{El engranaje del Plan FinEs desde la perspectiva de sus estudiantes egresa- dos/as}

Sin desconocer los múltiples rasgos que adopta el formato en el que se desarrolla la política, se focaliza su análisis en tres aspectos: la organización del tiempo, la organización de los espacios y la administración de los recursos. En relación con la organización del tiempo, se problematiza la noción de "flexibilidad" que plantea el Programa en diálogo con las experiencias de los/las estudiantes. En cuanto a la organización de los espacios, el análisis se realiza en dos planos: el de la puesta en marcha del FinEs a nivel jurisdiccional y en el marco de las diversas propuestas de tutorías. Por último, en el abordaje de la administración de los recursos, se presentan los materiales que el Programa pone a disposición en las sedes para la construcción de propuestas pedagógicas innovadoras y se analizan los modos en que estos aparecen (o no) en las propuestas de los/as docentes tutores desde la mirada de los/as estudiantes.

\section{La organización del tiempo}

Numerosos autores que problematizan la organización del tiempo en las instituciones educativas señalan que las jornadas escolares aún mantienen una estructura rígida basada en una concepción técnico-racional del tiempo, con unidades de duración uniformes y predefinidas (Escolano Benito, 2000). Así, el denominado módulo de clase sirve indistintamente para la enseñanza de diversos espacios curriculares y puede aplicarse en todos los niveles educativos. A partir de estas definiciones, surgen interrogantes como los siguientes. ¿El FinEs "rompe" con la estructura organizacional del tiempo de las jornadas escolares en las que los/las estudiantes transitaron sus vivencias previas? ¿ En qué medida los encuentros de tutorías proponen una organización novedosa del tiempo?

Tal como se ha mencionado, las normativas que regulan el Programa a nivel nacional y provincial definen el Plan como una propuesta educativa flexible, destinada a aquellos sujetos jóvenes y adultos mayores de 18 años que no lograron finalizar su último año de estudio en la escuela media. Los documentos consultados hacen referencia a la organización del tiempo en relación con la extensión del Programa:

El docente tutor deberá acordar con el alumno el Plan y Cronograma de producción de actividades de enseñanza y aprendizaje a lograr en 8 (ocho) encuentros tutoriales. El cumplimiento de esta agenda didáctica deberá ser flexible, reajustándose según avances y/o retrocesos. (Ministerio de Cultura y Educación, 2008, Anexo I: 3) 
Como puede observarse, se especifica la cantidad de encuentros tutoriales pero no su extensión. En las entrevistas, los estudiantes señalaron que los encuentros en el FinEs duraban 60 minutos, eran una vez por semana y requerían un porcentaje mínimo de asistencia obligatoria. Aquí es relevante mencionar la forma en que la normativa de la provincia de La Pampa se refiere a la asistencia. Mientras que la normativa nacional la describe como optativa, en esta provincia - tal como lo sostienen los/las estudianteses concebida como requisito fundamental para la aprobación de los espacios curriculares. A saber:

La participación a los ocho encuentros determinará en el alumno su condición de regularidad. Cuando un alumno, por razones justificadas, se vea imposibilitado cumplir con los ocho encuentros presenciales el tutor deberá sugerir actividades de aprendizaje de carácter compensatorio. (Ministerio de Cultura y Educación, 2008, Anexo II: 2)

El requerimiento aparece de forma reiterada en los discursos de los/las estudiantes de ambas sedes:

En Secretaría me dijeron que tenía que tratar de no faltar porque eran pocas clases y podía quedarme libre. (Entrevista Nº 2: 3; Sede General Pico)

Una vez llegué un poco tarde y ya me habían puesto el ausente [...] en todas las clases tomaron asistencia así en una planilla como en la escuela. (Entrevista $N^{\circ}$ 5: 2; Sede General Pico)

Cuando llegábamos lo primero que hacían los profesores era tomar asistencia, nunca nos dijeron de forma así directa que no íbamos a aprobar si faltábamos pero sabíamos que era importante ir. (Entrevista No 5 : 3; Sede Eduardo Castex)

Eran re pocas clases [...] es como que si no íbamos era lógico que no nos aprobaran... a una chica le pasó, fue una vez y dejó [...] igual podíamos arreglar los horarios con los profes para no perder clases. (Entrevista $\mathrm{N}^{\circ}{ }_{1}$ : 4; Sede Eduardo Castex)

Para los/as estudiantes, la asistencia es percibida como "necesaria" para la aprobación de los espacios curriculares y las acciones del personal de secretaría y de los docentes tutores refuerzan esta idea. A partir de estos datos, se advierte que en el Programa, en las sedes estudiadas, las posibilidades de aprendizaje se encuentran estrechamente asociadas con la presencialidad. Los y las jóvenes deben asistir a la mayor cantidad posible de encuentros de tutorías para conseguir una experiencia educativa exitosa. De este modo, en palabras de María Teresa González González (2010), el cumplimiento de la asistencia es leído por la política educativa como indicio de "implicación", de "enganche" y compromiso de parte del estudiante hacia su propio aprendizaje.

En las entrevistas, además, se consultaron las valoraciones de los/as egresados/as acerca de la cantidad y duración de los encuentros de tutorías. La mayoría expresó que si bien ocho encuentros fueron insuficientes para el abordaje de los temas de los espacios curriculares, la duración de cada uno de ellos fue acertada.

En lo que atañe a la organización de los horarios, se advierten algunas diferencias. Mientras que para quienes egresaron de la sede de General Pico estos eran impuestos y con muy poco margen de cambio, los/as estudiantes de la sede de Eduardo Castex destacan la flexibilidad de los/as docentes tutores/as para acordarlos.

A continuación, se citan algunas voces que reflejan las diferencias mencionadas: 
Estaba muy bueno el tema de los horarios. Los profesores nos preguntaban cuándo podíamos ir y si podían se acomodaban a nuestro horario. (Entrevista $\mathrm{N}^{\circ} 4$ : 5; Sede Eduardo Castex)

Me acuerdo que los profesores nos preguntaron cuándo podíamos ir a las clases antes de poner un horario... igual por ahí cuando se nos complicaba para ir a la mayoría lo cambiábamos de una semana a la otra. Lo importante era no perder la clase. (Entrevista $\mathrm{N}^{\circ}$ 5: 5; Sede Eduardo Castex)

Yo tuve que pedir permiso en el trabajo para llegar a horario a la escuela, para retirarme un rato antes. Pregunté si había posibilidad de cambiar los horarios y me dijeron que no, que los horarios eran fijos. (Entrevista $N^{\circ}$ 1: 4; Sede General Pico)

Como la escuela a la que íbamos tenía ocupada todas las aulas de mañana y de tarde, no nos quedaba otra que ir a la noche... (Entrevista $N^{\circ} 3$ : 3; Sede General Pico)

Estos testimonios hacen referencia a las posibilidades (en el caso de la sede de Eduardo Castex) e imposibilidades (en el caso de la sede de General Pico) de acordar los tiempos de aprendizaje de acuerdo a las obligaciones laborales y familiares fuera del Programa. Mientras que las experiencias los/as asistentes a la sede de Eduardo Castex suponen rupturas con el formato del Nivel Polimodal, las vivencias de los/as egresados/as de la sede de General Pico advierten fuertes continuidades. Vale señalar que en ambos casos se trata de sujetos que cuentan con experiencias de vida y tiempos de estudio que difieren cualitativa y cuantitativamente de las que presentan quienes asisten a una escuela secundaria común.

González González (2010) sostiene que las condiciones organizativas pueden generar un entorno propicio para los aprendizajes o, por el contrario, contribuir a un desajuste cotidiano entre estos y el funcionamiento del orden institucional propuesto. En los casos presentados, mientras que la flexibilidad en la organización de los encuentros es valorada de manera positiva por los/las estudiantes de la sede de Eduardo Castex (y resulta concordante con la normativa); las vivencias de los/as egresados/as de la sede de General Pico denotan, en términos de la autora, un "desajuste cotidiano" entre los propósitos que sustenta la política educativa y su materialización.

\section{La organización de los espacios}

La organización de los espacios constituye uno de los componentes básicos de la vida institucional. Blanca Benítez (2006) explica que las representaciones que los actores tienen sobre el espacio y cómo se sienten allí, condicionan con fuerte intensidad el trabajo y las vivencias en las instituciones. En esta línea, Estanislao Antelo (2007) sostiene que existen diversas formas de percibir el espacio. Una primera versión lo define como un espacio cerrado donde es posible localizar prácticas coercitivas como encerrar, clausurar, recluir, reprimir, aislar. Todas ellas evocan mecanismos o procedimientos de control cuyo objetivo es prohibir, impedir y bloquear. Una segunda mirada lo muestra con la función de refugio donde las prácticas parecen ser otras: asistencia, afecto, protección, seguridad, abrigo. El espacio pedagógico se convierte en un lugar donde el estudiante se siente acompañado.

A partir de estas conceptualizaciones, se indagó acerca de la construcción de los espacios en dos planos: uno vinculado a la puesta en acto del Programa en la provincia y otro relacionado con su organización en las sedes, como contexto de las propuestas de enseñanza y de aprendizaje. 
Como se anticipó, una de las normativas nacionales sostiene que el Programa podrá ponerse en funcionamiento en múltiples espacios. A saber:

Organización de las sedes

Las sedes funcionarán en:

- Entidades sindicales, empresariales y de la comunidad, que suscriban convenios con el Ministerio de Educación de la Nación y los Ministerios de las jurisdicciones.

- 2000 Escuelas secundarias comunes y de educación técnica que forman parte del Programa Nacional de Becas Estudiantiles.

- Escuelas secundarias de la modalidad de jóvenes y adultos (CENS). (Ministerio de Cultura y Educación; 2008, Anexo l: 1)

Como sostiene Plesnicar, en la definición de las sedes "podríamos advertir la apelación a la intersectorialidad y a la participación social en los procesos educativos casi como una rémora de las políticas y dinámicas de la Educación de Jóvenes y Adultos en nuestro país" (2014: 3). En la Provincia el Plan se desarrolla solo en escuelas, y en el Anexo I de una de las Resoluciones ya citadas incluso se detalla la nómina de instituciones que incluirán en sus espacios el Programa. ${ }^{4}$ De este modo, consideramos que en la provincia se escolariza la política, se propone para los/las estudiantes una experiencia educativa contextualizada en espacios con lógicas de acción conocidas por ellos/as.

Los autores citados al comienzo de este apartado advertían la fuerte incidencia de los espacios en la vida institucional de los sujetos. En diálogo con sus afirmaciones, surgen interrogantes como si la continuidad descripta habrá impactado de algún modo en las vivencias de los/as estudiantes. ¿Los sitios pensados para el desarrollo del Programa fueron siempre los mismos o se propusieron situaciones de aprendizajes en otros contextos? Aquí se observan nuevas diferencias entre las experiencias en las distintas sedes.

En los discursos de los egresados de la sede de General Pico, aparece el aula como el espacio privilegiado para el desarrollo de los encuentros de tutorías. Dos de los egresados lo expresan del siguiente modo:

Casi siempre estábamos en algún aula. Creo que a la biblioteca fuimos solamente una vez a ver un video... después era totalmente normal como en la escuela... (Entrevista $\mathrm{N}^{\circ}$ 4: 3; Sede General Pico)

En Física fuimos una vez al laboratorio pero después estábamos en el aula. Teníamos un cuadernillo con ejercicios que hacíamos usando el pizarrón, nada raro. (Entrevista $N^{\circ}$ 1: 2; Sede General Pico)

Estos dichos dan cuenta de experiencias transitadas en espacios conocidos. En el imaginario de uno de ellos es "normal" el uso del aula, lo cual remite a todas sus vivencias escolares previas. En el otro testimonio aparece un elemento que caracterizó desde sus inicios las prácticas escolares: el uso del pizarrón. Francois Dubet y Danilo Martucchelli (1998) sostienen que los sujetos construyen sus experiencias desde los inicios de su escolarización como estrategas autónomos, pero que muchas veces tal autonomía se encuentra limitada por las oportunidades que le ofrecen, los recursos que dispone y las exigencias del contexto. Así, el sujeto se convierte en un "gestionador de 
la incertidumbre" que debe adecuarse a los requerimientos de otros, en este caso los docentes, y pensar lógicas de acción contextualizadas. Si se retoman los testimonios de los/as estudiantes, vemos que estos/as se adecuan a los espacios que los/as docentes tutores/as proponen, construyen sus experiencias en esos "límites" sin cuestionamientos.

Por su parte, los/as estudiantes de la sede de Eduardo Castex resaltan la utilización de nuevos espacios. Expresan que las clases no solo se llevaban a cabo en las aulas, sino que también podían consensuarse momentos de consulta con el docente tutor en otros horarios y espacios que a veces podían exceder la misma institución educativa. Tres estudiantes relataron sus experiencias del siguiente modo:

Estaba bueno porque los profesores me hacían anotar otros horarios donde estaban libres en la escuela para pasar por cualquier duda... o cuando éramos muchos los que no podíamos ir, cambiábamos el horario a la noche. (Entrevista $\mathrm{N}^{\circ} 4$ : 5 , Sede Eduardo Castex)

Cuando faltábamos, la profesora de Filosofía nos daba la posibilidad de juntarnos en la biblioteca a la mañana los días que ella estaba. Yo nunca fui pero una de las chicas sé que sí. (Entrevista N²: 4; Sede Eduardo Castex)

Me acuerdo que uno de los profesores hasta nos pasó la dirección de su casa para ir antes de la última prueba... con uno de los chicos fuimos y nos sacamos las dudas que teníamos... había confianza. (Entrevista № 6: 7; Sede Eduardo Castex)

En las primeras líneas de este apartado, fueron explicadas las "funciones" que pueden tener los espacios desde la perspectiva de Antelo (2007). Se expuso que pueden generar prácticas coercitivas como recluir, aislar, reprimir; o aparecer como sitios de refugio, donde es posible el afecto, la protección, el abrigo. En diálogo con estos conceptos, se advierte que los/as estudiantes de la sede de Eduardo Castex hacen referencia a los espacios de encuentro desde la última perspectiva. Describen el Programa como una alternativa que deja atrás los recuerdos de las rígidas estructuras de tiempos y espacios que vivenciaron durante su paso por el Nivel Polimodal. A continuación, se citan otras voces al respecto:

En el FinEs era todo diferente de la escuela... como éramos poquitos en Filosofía, la profesora se sentaba al lado nuestro [...] podíamos preguntar lo que sea y no te daba vergüenza leer ni nada... Aunque vimos todos los temas muy rápido, me pareció bueno que fuésemos poquitos. (Entrevista № 2: 5; Sede Eduardo Castex)

El profesor de Economía, cuando llegábamos a la clase, nos hacía poner en ronda... Corregíamos las tareas entre todos y el después nos seguía explicando en el pizarrón... (Entrevista N ${ }^{\circ}$ 6: 4; Sede Eduardo Castex)

En las clases de Economía podíamos tomar mate mientras el profesor nos explicaba... En la escuela me acuerdo que no nos dejaban comer ni tomar nada. (Entrevista $\mathrm{N}^{\circ} 4$ : 7; Sede Eduardo Castex)

Hablar de la organización del espacio, entonces, no solo implica la consideración del medio físico sino de las interacciones que este posibilita o dificulta. En las entrevistas, los/as egresados/as valoran de manera positiva la ruptura de la clásica relación espacial asimétrica entre docente y estudiante, característica de las formas de arquitectura panópticas donde el docente se ubica frente al grupo de estudiantes (Escolano Benito, 2000). De este modo, ingresan escenas en las cuales es posible "estar en ronda" y "tomar mate". Para Elisabeth Bautier et al. (2000), se trata de interdependencia en la interacción entre docentes y alumnos. Esta remite a la medida en que la institución (en este caso 
la sede) y sus actores reconocen la necesidad de no presuponer que los/as estudiantes cuentan con las disposiciones y formas de vincularse con el conocimiento que "se supone" que deberían tener, sino que toman como punto de partida los saberes con los que efectivamente llegan.

Acordamos con Miguel Santos Guerra (2007) cuando plantea la necesidad de promover una adaptación de los espacios a las exigencias de la comunicación didáctica. Para el autor, el trabajo individual, la actividad en grupos, entre otras, requieren espacios diversos y flexibles. La rigidez en la utilización de los mismos dificulta el trabajo interdisciplinar y el aprendizaje globalizado. En esta línea, se considera que para los estudiantes de la sede de General Pico los espacios no fueron facilitadores de prácticas de enseñanza alternativas a las desplegadas por los/as docentes en sus trayectorias escolares previas. El predominio del aula condujo a formas rígidas de utilización de los espacios. En contraposición, en las experiencias de los/as egresados/as de Eduardo Castex, se observa la aparición de nuevas significaciones, los espacios son percibidos como múltiples y flexibles y son facilitadores de prácticas educativas diversas.

\section{La administración de los recursos}

En este apartado se analizan los recursos utilizados por los/as docentes tutores para llevar adelante sus propuestas pedagógicas. Interesa indagar la administración de estos a partir de las valoraciones construidas por los/as estudiantes acerca de su disponibilidad y utilización.

Edith Litwin (2009), en uno de sus aportes referido a las condiciones y los contextos que inciden en la conformación de las propuestas de enseñanza, explica que cualquiera sea el dispositivo o ayuda elegida por el docente, los recursos siempre se articulan con los propósitos en los que se inscribe la acción, la concepción de sujeto de aprendizaje y la modalidad de enseñanza en la que se inserta. A partir de ello, surgen interrogantes como estos: ¿de qué modos se articularon los recursos propuestos por el Programa con sus propósitos? ¿Posibilitaron la construcción de experiencias de aprendizaje alternativas, diferentes a las vivenciadas por los sujetos jóvenes y adultos en el Nivel Polimodal?

El análisis de las normativas que regulan el FinEs permite advertir que el Plan propone una "modalidad de enseñanza" donde los recursos aparecen diferenciados según su destinatario: estudiante, docente tutor y escuela sede. En el caso de los/as estudiantes, se detalla una serie de recursos disponibles dentro y fuera de los marcos institucionales; para los/as docentes tutores/as, se menciona la elaboración de "materiales de apoyo para la enseñanza y el aprendizaje", $\mathrm{y}$ en el caso de las escuelas sedes, se asegura el envío de materiales de lectura para el fortalecimiento de sus bibliotecas y se aclara que las mismas deberán poner a disponibilidad del Programa sus recursos físicos. Los documentos explicitan que la organización propuesta pretende responder a las necesidades educativas de los sujetos jóvenes y adultos en pos de favorecer aprendizajes valiosos y contextualizados.

En el caso de los/as estudiantes, una de las resoluciones nacionales ya citada en este trabajo explicita:

Para poder preparar las materias los estudiantes contarán con: 
- Teleclases del Canal Encuentro

- Plataforma virtual a través del Portal Educ.ar

- Libros de texto provistos por el Ministerio de Educación de la Nación

- Material de la Campaña Nacional de Lectura

- Biblioteca de la escuela sede del Plan FinEs

- Tutorías en las escuelas sedes del Plan FinEs

(Ministerio de Cultura y Educación; 2008, Anexo I: 2)

Como puede observarse, se mencionan dos recursos multimediáticos para su inclusión en las propuestas de enseñanza: Teleclases del Canal Encuentro y Plataforma virtual a través del Portal Educ.ar, acorde con el planteamiento de que los sujetos pueden aprender sin necesidad de asistir a todos los encuentros de tutorías. También se citan otros recursos como libros de texto y materiales de la Campaña Nacional de Lectura; las bibliotecas de las escuelas sedes y, por último, los encuentros de tutorías que -como ya se ha mencionado- en la provincia aparecen como requisito indispensable para la aprobación de los espacios curriculares.

En las entrevistas realizadas, los/as estudiantes se refieren a la utilización de solo dos de los recursos mencionados por la normativa: las bibliotecas y los libros de inglés. En sus testimonios, los recursos propios de la escuela como el laboratorio y la biblioteca son usados según la disponibilidad horaria de las instituciones educativas, situación que dificulta su inclusión en las propuestas de enseñanza planificadas por los/as docentes tutores/as.

A continuación, se citan algunas voces al respecto:

Nunca fuimos a la biblioteca, en Filosofía le dijimos a la profe de ver una película ahí pero por falta de tiempo nos dijo que no. Además, no siempre había alguien que abra la biblioteca... (Entrevista № 2: 6; Sede Eduardo Castex)

A veces íbamos al laboratorio, cuando estaba la llave (risas)... si no se podía nos quedábamos en el aula y hacíamos las actividades. (Entrevista $N^{\circ} 4$ : 3; Sede Eduardo Castex)

\footnotetext{
... al laboratorio solamente se podía ir de mañana... no sé por qué... supongo que porque en ese horario estaban los chicos del secundario. (Entrevista N 5: 5; Sede General Pico)

En Inglés usábamos a veces unos libros que había en la escuela... cuando estaba abierta la biblioteca, si no usábamos el cuadernillo que teníamos desde el principio. (Entrevista $N^{\circ}$ 1: 3; Sede General Pico)
}

Estos dichos permiten advertir ciertos desajustes entre el planeamiento del Programa y su concreción. En las sedes analizadas, los recursos disponibles son solo los proporcionados por las escuelas en las que se desarrolla el FinEs. Se considera que ello contribuye a replicar experiencias de aprendizaje similares a las vivenciadas por los estudiantes en el Nivel Polimodal.

Philippe Meirieu (2001), en su análisis de los aspectos instrumentales de la enseñanza, advierte la necesidad de preguntarnos si las herramientas propuestas -en este caso en el 
FinEs- sirven realmente para las finalidades que se pretende conseguir y si posibilitan la movilización de conocimientos. Aun cuando en el estudio no se analizaron las percepciones de los docentes tutores del Programa, los discursos de los estudiantes permiten advertir que los recursos efectivamente incluidos en el desarrollo de las propuestas de tutorías no se corresponden con los recursos disponibles enunciados en las normativas.

En términos de Florencia Finnegan (2016), esto puede comprenderse en el marco del carácter subalterno que aún tiene la EDJA pese a los significativos cambios que plantea la Ley de Educación Nacional. En relación con la administración y disponibilidad de recursos, la autora afirma:

Entre las deudas de esta década, aunque con significativas diferencias entre

jurisdicciones, se cuenta una falta de fortalecimiento de las ofertas escolares por la vía de la provisión de recursos y la transformación de los regímenes académicos, “flexibilidad" lograda las más de las veces a partir de arreglos de las propias instituciones. (Finnegan, 2016: 19; las comillas pertenecen al original)

La autora agrega que, si bien se contempló la modalidad de Jóvenes y Adultos en la provisión de libros, no fue incluida en el Programa Conectar Igualdad para la entrega de netbooks, situación que dificulta y limita el acceso a los recursos multimediáticos propuestos por las normativas del FinEs: teleclases del Canal Encuentro y plataforma virtual del Portal Educ.ar, entre otros.

\section{A modo de cierre}

Las observaciones realizadas en este artículo en torno al formato organizacional del Programa permiten conocer -al menos en principio- cómo los actores de las sedes organizan los tiempos, definen los espacios y administran los recursos a partir de las condiciones institucionales en las que se pone en funcionamiento el Plan y las representaciones y significaciones que los sujetos construyen en torno a los textos de la política. La previsión medios-fines aparece como rasgo común en las sedes y se vincula con los modos en que el Programa es recepcionado en el contexto de la práctica (Ball, 2008). Los rasgos centrales del formato organizacional, que dan origen al Programa y se plasman en su diseño macropolítico, son resignificados/reinterpretados por quienes toman decisiones a nivel jurisdiccional, y nuevamente interpretados/traducidos por los actores locales (directivos, docentes, estudiantes), quienes a partir de los recursos disponibles materializan la política en las sedes. En el caso de la sede de General Pico, presenta límites para el desarrollo de la "agenda didáctica flexible"; y en el caso de la sede de Eduardo Castex, parece promover experiencias de aprendizajes valoradas de manera positiva por los/as estudiantes.

Los análisis del formato organizacional del Programa llevan a reflexionar, además, en torno al concepto de "flexibilidad" que emerge con fuerza en esta y otras políticas educativas en los últimos años. Mercedes Saccone (2016) señala que en los programas estatales posteriores al año 2001 predomina una tendencia a la flexibilización de los aspectos centrales que hacen a su concreción y que ello es concebido como factor central para conseguir la inclusión educativa y con ella la inclusión social de los y las jóvenes. Sostiene que los aspectos que se desean modificar pueden agruparse en dos áreas: "administrativa y de gestión" y "curricular y pedagógica". Los aspectos del formato del FinEs analizados en este artículo pueden comprenderse en el marco del primer grupo, aunque con fuertes incidencias en el segundo. 
Si bien el Programa plantea una propuesta pedagógica autodefinida como "innovadora" y adaptada a las necesidades de los/as jóvenes y adultos que no pudieron terminar la escuela secundaria, se materializa "entre lo nuevo y lo viejo". Esto es, mantiene muchos de los rasgos propios del formato organizacional de la escuela secundaria tradicional, que combina con intentos de cambios e innovación. Esto cobra especial relevancia cuando se trata de sujetos que llegan al programa luego de vivenciar experiencias de fracaso en el nivel, como es el caso del FinEs.

La escolarización de la política conduce a problematizar los modos en los cuales el Estado da respuestas a las necesidades y demandas de los sujetos jóvenes y adultos en el contexto provincial. La flexibilidad de las propuestas pedagógicas en el marco de condiciones institucionales precarias (límites en la disponibilidad de espacios y recursos materiales y humanos) corre el riesgo de convertir la política en un circuito de mera acreditación formal, alejado de los propósitos de inclusión educativa y social pregonados en su normativa.

A más de una década de la sanción de la Ley de Educación Nacional $N^{\circ} 26206$, y en el marco de los avances en materia de políticas de inclusión promovidas en la Modalidad de Educación Permanente de Jóvenes y Adultos, se considera que todavía queda un largo camino para conseguir equiparar la igualdad de oportunidades en el acceso a la escolarización con la igualdad en el acceso a los bienes culturales por parte de los/ as jóvenes. Pareciera que aún no surgen políticas que apunten a una transformación integral de la escuela secundaria en la Argentina. 


\section{Bibliografía}

"Antelo, E. (2007). Variaciones sobre el espacio escolar. En Baquero, R.; Diker, G. y Frigerio, G. (Comps.). Las formas de lo escolar. Buenos Aires: Del Estante, pp. 59-78.

" Ball, S. (1997). Sociología política e investigación social crítica: una revisión de las políticas educativas actuales. Revista Británica de Investigación Educativa, 23(3), pp. 257-274.

»Ball, S. (2002). Textos, discursos y trayectorias de la política: la teoría estratégica. Páginas de la Escuela de Ciencias de la educación, 2 (2 y 3), pp. 19-33.

"Ball, S. (2008). Herramientas analíticas para interpretary debatir las políticas educativas. Bristol: University of Bristol.

» Baquero R., Diker, G. y Frigerio, G. (2007). Las formas de lo escolar. Buenos Aires: Del Estante.

" Bautier, E.; Charlot, B. y Rochex, J. (200o). Entre apprentissages et métier d'élève: le rapport au savoir. En Van Zanten, A. (Dir.). L'école. L'etat de savoirs. París: Centre National du Livre, pp. 179-188.

"Benítez, B. (2006). Los espacios escolares. En Brandi, L. S. (Dir.). Conocimiento escolar y Cultura Institucional. La transposición del conocimiento en circuitos escolares diferenciados. Buenos Aires: Miño y Dávila, pp. 127-138.

»Dubet, F. y Martuccelli, D. (1998). En la escuela. Sociología de la experiencia escolar (1 $1^{\underline{a}}$ ed.). Buenos Aires: Losada.

»Dussel, I. (2006). Currículum y Conocimiento en la escuela media argentina. Revista Anales de la Educación Común, 4 (8), pp. 95-105.

»Escolano Benito, A. (200o). Tiempos y Espacios para la escuela. Ensayos Históricos. Madrid: Biblioteca Nueva.

» Finnegan, F. y Brunetto, C. (diciembre, 2014). "Acá hay un trabajo político": Actores y relaciones en la producción del Plan FinEs 2 Secundaria en la Provincia de Buenos Aires. (Ponencia). VIII Jornadas de Sociología de la Facultad de Humanidades y Ciencias de la Educación. Universidad Nacional de La Plata, Ensenada, Argentina.

» Finnegan, F. (2016). Educación Permanente para Jóvenes y Adultos. Alto incremento de la matrícula. En 10 Años de la Ley de Educación Nacional. Buenos Aires: UNIPE Editorial Universitaria, pp. 19-20.

»González González, M. T. (2010). El alumno ante la escuela y su propio aprendizaje: Algunas Líneas de investigación en torno al concepto de Implicación. Revista Iberoamericana sobre Calidad, Eficacia y Cambio en Educación, 4(8), pp. 11-31.

» Litwin, E. (2009). El oficio de enseñar. Condiciones y contextos. Buenos Aires: Paidós.

" Mainardes, J. y Marcondes, M. (2009). Interview with Stephen J. Ball: A dialogue about social justice, research and education policy. Revista Educação y Sociedade (5), pp. 303-318.

» Meirieu, P. (2001). La opción de educar. Ética y Pedagogía. España: Octaedro.

" Ministerio de Cultura y Educación, Dirección General de Educación Polimodal y Superior. Sobre la implementación del Plan FinEs en la Provincia de La Pampa; Expediente $\mathrm{N}^{\circ} 10129 / 08$.

» Ministerio de Cultura y Educación (2008). Dirección General de Educación Polimodal 
y Superior. Sobre la implementación del Plan FinEs en la Provincia de La Pampa. Expediente $\mathrm{N}^{\circ}$ 10129/08.

» Ministerio de Cultura y Educación (2008). Plan de Finalización de Estudios Primarios y Secundarios para Jóvenes y Adultos. Presentación y Líneas de Acción 2008- 2011. Recuperado de: <http://www.oei.es/pdfs/plan_fines_argentina.pdf>

» Ministerio de Educación de la Nación Argentina. Consejo Federal de Educación. Recuperado de: <http://www.lapampa.gov.ar/images/stories/Archivos/AsesoriaLetrada/ Leyes/2008/Ley_2462.pdfs

» Ministerio de Educación de la Nación. Consejo Federal de Educación. Resolución № 66/o8. Recuperado de: <http://www.me.gov.ar/consejo/resoluciones/reso8/66-o8.pdfs

»Neiman, G. y Quaranta, G. (2007). Los estudios de caso en la investigación sociológica. En Vasilachis de Gialdino, I. (Coord.). Estrategias de investigación cualitativa. Buenos Aires: Gedisa, pp. 213-237.

"Plesnicar, L. (noviembre, 2014). Políticas educativas y educación secundaria en la Argentina. El FinEs como estrategia de inclusión educativa. (Ponencia). I Bienal Latinoamericana de Infancias y Juventudes. Democracias, Derechos Humanos y Ciudadanías. Manizales, Colombia.

» Saccone, M. (2016). La educación media en tiempos de transformaciones: una mirada socio-antropológica hacia México y Argentina. Buenos Aires: Cuadernos CLACSOCONACYT.

»Santos Guerra, M. (2007). Espacios escolares. Cuadernos de Pedagogía (217), pp. 55-64.

»Southwell, M. (2010). La educación secundaria en la Argentina. Notas sobre la historia de un formato. En Tiramonti, G. (Dir.). Variaciones sobre la forma escolar. Límites y posibilidades de la escuela media. Rosario: Homo Sapiens, pp. 35-69.

»Tenti Fanfani, E. (2016). ¿Derecho al conocimiento o derecho a la escolaridad? En 10 Años de la Ley de Educación Nacional. Buenos Aires: UNIPE Editorial Universitaria, pp. 7-9.

"Terigi, F. (mayo, 2007). Los desafíos que plantean las trayectorias escolares. (Ponencia). III Foro Latinoamericano de Educación Jóvenes y Docentes. La escuela secundaria en el mundo de hoy. Buenos Aires: Fundación Santillana.

"Terigi, F. (2009). Las trayectorias escolares: del problema individual al desafío de política educativa. Proyecto Hemisferio "Elaboración de Políticas y Estrategias para la Prevención del Fracaso Escolar”. Organización de los Estados Americanos (OEA)/ Agencia Interamericana para la cooperación y el desarrollo. Buenos Aires: Ministerio de Educación de la Nación.

» Tiramonti, G. et al. (2007). Nuevos formatos escolares para promover la inclusión educativa. Un estudio de caso: la experiencia argentina. Buenos Aires: FLACSO.

» Trilla, J. (1985). Ensayos sobre la escuela. El espacio social y material de la escuela. Barcelona: Laertes.

»Ziegler, S. (2011). Entre la desregulación y el tutelaje: ¿hacia dónde van los cambios en los formatos escolares? En Tiramonti, G. (Dir.). Variaciones sobre la forma escolar. Límites y posibilidades de la escuela media. Buenos Aires: Homo Sapiens, pp. 71-88.

\section{Mariana Correa}

Profesora y Licenciada en Ciencias de la Educación (Facultad de Ciencias Humanas, Universidad Nacional de La Pampa). Becaria doctoral, Consejo Nacional de Investigaciones Científicas y Técnicas, Universidad Nacional de La Pampa, Argentina. profmarianacorrea@gmail.com 